\section{P2-156 THE VULNERABLE ELDERS SURVEY (VES-13) AS A SCREENING INSTRUMENT IN ELDERS OF BRAZILIAN LONG-STAY INSTITUTIONS}

doi:10.1136/jech.2011.142976i.91

L L Luz, ${ }^{*}$ L M Santiago, I E Mattos. National School of Public Health, Oswaldo Cruz Foundation, Rio de Janeiro, RJ, Brazil

Introduction The Comprehensive Geriatric Assessment (CGA) is an evaluation conducted on older people to detect limitations of health in multiple domains. However, CGA is a time-consuming assessment and so the use of abbreviated screening instruments, as the Vulnerable Elders Survey (VES-13) has been proposed. The purpose of this study was to evaluate the performance of the VES-13 in elders who live in Brazilian long-stay institutions.

Methods This is a study with elderly residents of long-stay institutions in four Brazilian cities. The assessment of functional, emotional and cognitive domains was performed with CGA and with VES-13. Individuals were scored separately with both instruments and classified accordingly. The proportion of positives identified in each domain with the CGA was compared to that of the VES-13. The characteristics of the targeted group were compared with those not selected using the $\chi^{2}$ test.

Results These are preliminary results for 340 elders. Mean age was 75.5 years and 304 (89.4\%) were considered as vulnerable (score $\geq 3$ ). VES-13 identified correctly $90.5 \%$ of the elders with cognitive impairment, $99.3 \%$ of those with dependence in $\mathrm{ADL}, 99.5 \%$ of those in IADL and $86.9 \%$ of those who had depression. Compared to those not selected targeted individuals were older ( 80 years or more), male, had cognitive impairment and were dependent in $\mathrm{ADL}$ and in IADL $(\mathrm{p}<0.001)$.

Conclusions VES-13 performed well as a screening instrument in this particular setting, identifying the majority of those elders with functionality problems, cognitive impairment and depression and who would need further evaluation with CGA.

\section{P2-157 MORTALITY TRENDS OF PROSTATE CANCER IN ELDER BRAZILIANS, 1980-2008}

doi:10.1136/jech.2011.142976i.92

${ }^{1} \mathrm{~L}$ L Luz, ${ }^{* 1} \mathrm{~L}$ M Santiago, ${ }^{1} \mathrm{I}$ E Mattos, ${ }^{1} \mathrm{D}$ B Ferreira, ${ }^{2,3} \mathrm{~J}$ F S da Silva. ${ }^{1}$ National School of Public Health, Oswaldo Cruz Foundation, Rio de Janeiro, Rio de Janeiro, Brazil; ${ }^{2}$ State of Mato Grosso do Sul Health Secretariat, Campo Grande, MS, Brazil, ${ }^{3}$ Federal University of Mato Grosso do Sul, Campo Grande, MS, Brazil

Introduction While incidence and mortality rates for most cancers are decreasing in developed countries, they are increasing in the less developed. Prostate cancer is becoming one of the most frequent cancers in men. The aim of this study was to analyse trends of mortality for prostate cancer in elder Brazilians.

Methods All deaths with prostate cancer as the underlying cause, occurring during 1980-2008, in the 27 Brazilian States, in men aged 60 years or more, were identified in the Mortality Information System, a population-based nationwide registry. Population data was obtained from the Brazilian Institute of Geography and Statistics. Trends were analysed through polynomial regression models, using adjusted and age-specific annual rates of mortality for each State.

Results Prostate cancer mortality has been increasing in all Brazilian States, although some showed a certain pattern of stability for the more recent years. There was considerable regional variability in the magnitude of the annual increments, varying from 1.30 to 6.25 deaths per 100000 men/year. Regarding trends by age groups, patterns were similar to those observed for the group as a whole.
Conclusion Incidence of prostate cancer has greatly increased over the last decades, following the advent of the prostate-specific antigen test. Consequently, interpreting temporal trends in mortality has become difficult. It remains unclear to what extent the increasing mortality rates are due to detection of the disease or are representing a rise in its incidence. The increase in mortality observed in Brazil requires further monitoring.

\section{P2-158 MORTALITY FROM HAEMATOLOGICAL MALIGNANCIES IN OLDER BRAZILIANS, 1996-2008}

doi:10.1136/jech.2011.142976i.93

L L Luz, * I E Mattos, D B Ferreira, L M Santiago. National School of Public Health, Oswaldo Cruz Foundation, Rio de Janeiro, Brazil

Introduction Incidence and mortality of haematological malignancies have been increasing in Brazil, as a great number of individuals reach the age of 60 years. The aim of this study was to analyse patterns of mortality from haematological cancers in older Brazilians.

Methods Deaths from haematological malignancies in individuals of 60 or more years in 11 States were identified in the Mortality Information System, a population-based nationwide registry. Population data was obtained from the Brazilian Institute of Geography and Statistics. Polynomial regression was used to analyse trends in age-adjusted and age-specific mortality rates.

Results Leukaemia showed the highest rates, varying from 11.96 to 17.58 per 100000 . Trends of increment were observed in three States, while a declining trend was observed in one. Mortality rates of Non-Hodgkin lymphoma ranged between 6.64 and 16.35 per 100000 and presented regional variability, with declining trends in South and Southeast States and increments in Central-West States. Mortality rates for myeloma showed steady increasing trends in four States. For Hodgkin's disease, declining trends were seen in two States. Trends of increment were observed for leukaemia and NonHodgkin lymphoma in the 80 or more age-group.

Conclusions The Southeast is the most industrialised Brazilian region with major petroleum exploitation and petroleum-based industries. The South and the Central-West are areas of extensive agriculture. Although the aetiology of these malignancies is still largely undefined, they have some common potential risk factors (solvents, pesticides) which could have contributed to the observed trends. These findings need further analysis to better characterise mortality patterns.

\section{P2-159 CAN THE INCREASED INCIDENCE OF MELANOMA BE EXPLAINED SOLELY BY ENHANCED SURVEILLANCE AND AWARENESS? A STUDY USING SECOND MELANOMA AS AN INDICATOR}

doi:10.1136/jech.2011.142976i.94

${ }^{1} \mathrm{~L}$ Liu, ${ }^{* 1,2} \mathrm{E}$ de Vries, ${ }^{2} \mathrm{~T}$ Nijsten, ${ }^{1,3} \mathrm{~J}$ W Coebergh, ${ }^{1} \mathrm{I}$ Soerjomataram. ${ }^{1}$ Department of Public Health, Erasmus University Medical Center, Rotterdam, The Netherlands, ${ }^{2}$ Department of Dermatology, Erasmus University Medical Center, Rotterdam, The Netherlands: ${ }^{3}$ Comprehensive Cancer Center South (IKZ), Eindhoven, The Netherlands

Background Melanoma incidence has nearly doubled in the past 2 decades in the Netherlands. It has been debated whether this sharp increase was mainly due to enhanced surveillance and awareness. If this were true then this trend would be even more significant among cancer patients.

Material and Methods Using data from the Netherlands Cancer Registry, we investigated the trend in the risk of developing melanoma among 1270815 cancers patients diagnosed between 1989 and 2008. Standardised incidence ratios (SIR) were computed to 
express melanoma risk among cancer patients as compared with the general population.

Results 4101 patients with a first invasive cancer were subsequently diagnosed with a melanoma. In the first year after diagnosis, risk of being diagnosed with melanoma was more than 10-fold higher among skin cancer patients as compared with the general population ( $\mathrm{SIR}=16.595 \% \mathrm{CI} 15.1$ to 17.9). The risk dropped to 6.9 (SIR) (95\% CI 6.4 to 7.5 ) 2-5 years post-diagnosis. After non-skin cancer, this drop is much less significant, from SIR $=1.6$ (95\% CI 1.4 to 1.7$)$ to $S I R=1.4(95 \%$ CI 1.3 to 1.5$)$ in $0-1$ years and $2-5$ years after first cancer, respectively. No trend of SIR during the study period was observed.

Discussion and Conclusion Intensive clinical check-up among skin cancer patients may explain the inflated risk of second melanoma, especially in the first year post-diagnosis. Our findings suggest that increased surveillance and awareness were not the sole explanation for the rising incidence of melanoma in the Netherlands.

\section{P2-160 RIGHT-SIDE SHIFTING OF SECOND COLORECTAL CANCER-IMPLICATIONS FOR AETIOLOGY AND CLINICAL RELEVANCE}

doi:10.1136/jech.2011.142976i.95

${ }^{1} \mathrm{~L}$ Liu, ${ }^{* 2} \mathrm{~V}$ Lemmens, ${ }^{3}$ de Hingh, ${ }^{1} \mathrm{E}$ de Vries, ${ }^{4} \mathrm{M}$ van Leerdam, ${ }^{1,2} \mathrm{~J}$ W Coebergh, II Soerjomataram. 'Department of Public Health, Erasmus University Medical Center, Rotterdam, The Netherlands; ${ }^{2}$ Comprehensive Cancer Center South (IKZ), Eindhoven, The Netherlands; ${ }^{3}$ Department of Surgery, Catharina Hospital, Eindhoven, The Netherlands; ${ }^{4}$ Department of Gastroenterology and Hepatology, and Internal Medicine, Erasmus University Medical Center, Rotterdam, The Netherlands

Background Colorectal cancer (CRC) is a heterogeneous disease: cancers in proximal, distal colon and rectum show differences in carcinogenesis pathways (proximal colon cancer related to microsatellite instability vs distal colon cancer to allelic losses), epidemiological patterns and clinical characteristics. By assessing the risk of second primary CRC among CRC patients, we aimed to shed light on the aetiology of multiple CRCs and its clinical relevance.

Material and Methods We analysed the risk of second CRC among 123253 first CRC patients from the Netherlands Cancer Registry data. Standardised incidence ratio (SIR) was computed to compare risk of second CRC among CRC patients with the general population.

Results During a median follow-up of 2 years, 2720 second CRC were diagnosed in CRC patients. More than $50 \%$ of second CRC were located in the proximal colon translating into a fourfold $R R$ when compared with general population (SIR=4.1, 95\% CI 3.9 to 4.3). Although we did not observe specific high risk pairs between sub sites of the first and second CRC, interestingly, right-side predomination of second CRC risk was clear. The right-side shifting of second CRC persists even after 10 years of follow-up in all patients' sub-groups that is, synchronous and metachronous cancers, by follow-up time and sub-sites of first CRC.

Discussion and Conclusion Our results highlighted the crucial role of microsatellite instability in the development second CRC. Due to the persistently elevated risk of a proximal colon cancer, surveillance on this specific site is recommended, preferably using FOBT.

\section{P2-161 INCREASED MELANOMA RISK AMONG MELANOMA PATIENTS}

doi:10.1136/jech.2011.142976i.96

${ }^{1} \mathrm{~L}$ Liu, ${ }^{*} \mathrm{R}$ van der Leest, ${ }^{3} \mathrm{~T}$ Nijsten, ${ }^{1,2} \mathrm{~J}$ W Coebergh, ${ }^{1,3} \mathrm{E}$ de Vries. ${ }^{1}$ Deparment of Public Health, Erasmus University Medical Center, Rotterdam, The Netherlands;
${ }^{2}$ Comprehensive Cancer Center South (IKZ), Eindhoven, The Netherlands; ${ }^{3}$ Department of Dermatology, Erasmus University Medical Center, Rotterdam, The Netherlands

Background Study on risk of subsequent melanoma among melanoma patients is scarce.

Objective To investigate melanoma risk in melanoma patients. Material and Methods Netherlands Cancer Registry incidence data (1989-2008) were employed. Patients $(\mathrm{N}=57817)$ diagnosed melanoma in this period were followed. Follow-up censored at second melanoma, death or end of study. Standardised incidence ratio (SIR) was used expressing RR compared to the general population. Sexand follow-up-specific SIR were computed under for both first and second in situ and invasive melanomas. Cox regression model which contains age at diagnosis, sex, Breslow thickness, and follow-up periods was constructed to estimate relative excessive risk melanoma risk.

Results During a median of 3 yrs of follow-up time, 1985 patients diagnosed for a second melanoma. After both in situ and invasive melanoma, inflated risk for a second melanoma was observed: after a first in situ melanoma, the risk to develop a subsequent melanoma was 16.3 (SIR), 95\% CI 14.7 to 17.9. Following an invasive melanoma, risk was 13.3 (SIR), 95\% CI 12.6 to 14.1. Elevated risks were observed in all follow-up periods $(0-1 \mathrm{yr}, 2-5 \mathrm{yr}, 6-10 \mathrm{yrs}$, $10-15$ yrs, $16-20$ yrs) in both sexes. Age at diagnosis, sex, Breslow thickness, were shown to explain the excess risk observed in those follow-up periods.

Discussion and Conclusion Based on the persistently high risk of melanoma among melanoma patients, a long-term follow-up schedule is needed for both patients and clinicians.

\section{P2-162 BONE ACCRUAL AT THE FOREARM DURING ADOLESCENCE: IS PREVENTION LIKELY TO WORK?}

doi:10.1136/jech.2011.142976i.97

\author{
${ }^{1,2} \mathrm{R}$ Lucas, ${ }^{*}{ }^{1,2} \mathrm{M}$ Severo, ${ }^{1,2} \mathrm{E}$ Ramos, ${ }^{1,2} \mathrm{H}$ Barros. ${ }^{1}$ Department of Hygiene and \\ Epidemiology, University of Porto Medical School, Porto, Portugal; ${ }^{2}$ Institute of Public \\ Health of the University of Porto, Porto, Portugal
}

Introduction Whether bone quality is essentially modifiable or tracks predictably up to adulthood is unclear. We aimed to identify a biological timing when interventions may have optimal impact on future bone properties.

Methods Participants were 709 girls from a cohort of adolescents born in 1990 and evaluated at 13 and 17 years-old. Evaluations consisted of structured questionnaires including gynaecologic age (GA) and physical evaluations comprising height, weight, body composition (bioelectric impedance) and bone mineral density (BMD) at the forearm (dual-energy x-ray absorptiometry). Our outcome was $\mathrm{BMD}$ variation during follow-up. We used linear regression to estimate its associations with baseline $\mathrm{BMD}$ and anthropometry, in four GA classes at baseline: $\leq-1,0,1$ and $\geq 2$ years relative to menarche.

Results Mean (95\% CI) annual BMD variation from 13 to 17 yearsold was highest in the earliest GA group $\left(0.030(0.028 ; 0.032) \mathrm{g} / \mathrm{cm}^{2}\right.$ year) and lowest in the oldest GA (0.018 (0.016; 0.019)). Pearson's correlation between baseline $\mathrm{BMD}$ and its increase varied from -0.12 in the youngest to -0.45 in the oldest GA. After adjustment (baseline $\mathrm{BMD}$, weight, fat mass and height) and standardisation, $\mathrm{BMD}$ variation in the lowest GA was associated with baseline BMD (-2.70 (-5.29; -0.108) per SD), but essentially with baseline weight $(11.4(0.523 ; 22.2))$ and fat mass $(-10.0(-19.2$; $-0.912)$ ). In the highest $\mathrm{GA}, \mathrm{BMD}$ variation was strongly determined by baseline $\mathrm{BMD}(-5.44(-7.20 ;-3.69))$ but not by anthropometric variables. 